\title{
Conservative Treatment of Dentigerous Cysts in Childhood
}

${ }^{1}$ Müge Çina Aksoy, ${ }^{2}$ Gülperi Koçer, ${ }^{3}$ Gülsün Yildirim, ${ }^{4}$ Timuçin Baykul, ${ }^{5}$ Doğan Dolanmaz

\begin{abstract}
Objectives: Developmental odontogenic jaw cysts are generally connected with impacted or unerupted teeth. The conservative treatment options of these types of cysts will be presented.
\end{abstract}

Study design: In this study 17 cases operated in Süleyman Demirel and Selcuk Universities Faculty of Dentistry were included. The data of the cases were documented with respect to age, sex, localization, symptoms, presence of unerupted teeth on panoramic radiographs. The cysts were treated with marsupialization with an obturator made of acrylic resin in nine patients and a tube drain in eight patients to keep the window open.

Results and conclusion: Marsupialization was evaluated as extremely effective in 15 patients, the cysts completely disappeared and the permanent teeth were erupted, in two patients the cysts disappeared but the teeth were not erupted.

Enucleation, marsupialization or their combination are the conventional treatment options of the jaw cysts. Large cysts may localize nearby the important anatomical structures or may involve unerupted permanent teeth. These kind of cysts may be treated with marsupialization in order to protect these anatomical structures or to allow the eruption of the permanent teeth.

Marsupialization should be considered as an effective conservative treatment method of the jaw cysts during mixed dentition in the childhood.

Keywords: Children, Conservative, Dentigerous cyst, Impacted teeth, Marsupialization.

How to cite this article: Aksoy MÇ, Koçer G, Yildirim G, Baykul T, Dolanmaz D. Conservative Treatment of Dentigerous Cysts in Childhood. Int J Experiment Dent Sci 2014;3(1):14-18.

Source of support: Nil

Conflict of interest: None declared

\section{INTRODUCTION}

Dentigerous cysts are the most common type of noninflammatory odontogenic cysts and are generally associated with

\footnotetext{
${ }^{1,2}$ Assistant Professor, ${ }^{3}$ Associate Professor, ${ }^{4,5}$ Professor

${ }^{1,2,4}$ Department of Oral and Maxillofacial Surgery, Faculty of Dentistry, Suleyman Demirel University, Isparta, Turkey

${ }^{3,5}$ Department of Oral and Maxillofacial Surgery, Faculty of Dentistry, Selçuk University, Konya, Turkey
}

Corresponding Author: Müge Çina Aksoy, Assistant Professor, Department of Oral and Maxillofacial Surgery Faculty of Dentistry, Suleyman Demirel University, Isparta Turkey, Phone: 902462118851, e-mail:mugecina@hotmail.com impacted or unerupted teeth. ${ }^{1-5}$ Follicular cysts frequently occur before the age of 20 years and their treatment can cause eruption problems, especially during eruption of the mixed dentition. ${ }^{1,3}$ Several long-term developmental processes occur in the maxillofacial area in pediatric age. These processes include the three-dimensional growth of the maxillofacial skeleton as well as odontogenesis of the deciduous and permanent dentition, all of which may be associated with cyst formation. ${ }^{6}$

Patients with these cysts do not typically experience pain. The most important features of these cysts are their ability to expand asymptomatically and their potential to displace adjacent teeth. ${ }^{1}$ The recommended treatments for jaw cysts include enucleation, curettage, decompression, and marsupialization. ${ }^{4}$ Removal of the associated impacted tooth and enucleation of the lesion are considered as a definitive treatment method of dentigerous cysts but marsupialization provides a less extensive and safe conservative form of treatment. ${ }^{6,7}$ Marsupialization and decompression all refer to creating a surgical window in the cyst wall and maintaining continuity between the cyst and the oral cavity.

This report presents a series of odontogenic cysts treated with marsupialization in order to allow the eruption of permanent teeth.

\section{MATERIALS AND METHODS}

This retrospective study included 21 cystic lesions (4 bilateral cases) in 17 patients (10 boys, 7 girls) with dentigerous cysts aged 6 to 14 years (mean (SD), $9 \pm 7,3$ years) who underwent surgery at Süleyman Demirel and Selçuk Universities Faculty of Dentistry, Department of Oral and Maxillofacial Surgery. The lesions were detected by clinical and radiographical examination, and the diagnoses of the lesions were confirmed by histopathological examination.

Of the total cases, six were asymptomatic and were diagnosed during routine radiographic examination. However, four cases were associated with dental caries, 8 with an intraoral swelling, two with infection and pain in the region, and 1 with a painful extraoral swelling.

Of the 21 lesions, 11 cysts occurred in the mandible: in the premolar region in five patients, the molar region in 1 patient, and the canine region in 1 patient. Three cysts were localized to the maxillary premolar region. The cysts occurred on both sides in four patients. 
The patient data were documented with respect to age, sex, localization, symptoms, and the presence of unerupted teeth on panoramic radiographs (2002 CC Proline, Planmeca, Finland, with 1:1.2 magnification). The diameters of the cystic lesions were measured radiographically.

The cysts were treated with marsupialization under local anesthesia (Ultracain DS forte, Aventis, İstanbul, Turkey) by the authors. To keep the window open after marsupialization, an acrylic resin obturator was used in nine patients and a tube drain in eight patients.

Marsupialization was continued until the eruption of the permanent teeth. The duration of marsupialization ranged from 6 to 24 months (mean 10.7 months).

\section{RESULTS}

Marsupialization was evaluated as extremely effective in 15 patients because the cysts completely disappeared radiographically and the permanent teeth erupted (Figs 1 to 3), whereas the size of the cysts were reduced but the cystic lesions became infected after marsupialization and the teeth did not erupt in two patients (Table 1). The selection of patients who would receive an acrylic resin obturator or a tube drain was random, and the results revealed no differences between these 2 groups.

\section{DISCUSSION}

The majority of cystic lesions of developmental origin that occur during childhood are associated with the continuous growth and subsequent changes in the dentition that take place until the end of puberty. ${ }^{8}$ In this study, all cases were diagnosed during childhood and all but one of the lesions were clinically and histopathologically diagnosed as dentigerous cysts. Only one case was diagnosed as a radicular cyst. The literature suggests that pericoronal radiolucencies larger than $2.5 \mathrm{~mm}$ in width are pathological and show cystic changes on histopathological analysis. ${ }^{9}$ All of the lesions were larger than $2.5 \mathrm{~mm}$ and were histopathologically diagnosed as cysts in this study.

Hyomoto et al reported that the most common sites of dentigerous cysts are the mandibular premolar and maxillary canine areas in the preadolescent patient. ${ }^{10}$ Only 3 of the lesions were localized in the maxilla and 18 were localized in the mandible in this study, and all lesions involved permanent teeth.

Enucleation, marsupialization, or a combination of these treatments is the conventional treatment option for jaw cysts. ${ }^{3,4,11}$ Marsupialization is a more conservative treatment model for jaw cysts and limits the extent of surgery. ${ }^{6,711}$ It was also more acceptable to the child and parents in addition to preventing any damage to the crowns of the developing permanent teeth. ${ }^{11}$

Marsupialization into the oral cavity involves the conversion of a cyst into a pouch by suturing the cyst lining to the oral mucosa or inserting a tube drain into the cyst cavity. This procedure results in decompression of the cystic lesion, ${ }^{4}$ allowing new bone to fill the defect. ${ }^{7}, 12$ The co-operation of the patient and parents was fundamental to the success of the treatment as they fully complied with the postoperative oral hygiene measures. ${ }^{11}$ After decompression, the cystic cavity slowly decreases in size. ${ }^{6}$ Large cysts may be localized close to important anatomical structures or may involve unerupted permanent teeth. These types of cysts can be treated with marsupialization in order to protect these anatomical structures or to allow the eruption of the permanent teeth. ${ }^{2-4,7,10-13}$ Marsupialization, which can lead to substantial reduction in the size of the cystic cavity,

Table 1: Patients and the results of marsupialization

\begin{tabular}{|c|c|c|c|c|c|c|}
\hline Cases & Gender & Age & Localization & Treatment & Eruption & Follow-up \\
\hline 1 & $\mathrm{M}$ & 9 & Mandibular premolar-bilateral & Marsupialization (tube drain) & Erupted & 6 months \\
\hline 2 & M & 14 & Mandibular premolar- right & Marsupialization (tube drain) & Erupted & 2 years \\
\hline 3 & $\mathrm{~F}$ & 6 & Mandibular premolar-bilateral & Marsupialization (tube drain) & Erupted & 1 year \\
\hline 4 & $\mathrm{~F}$ & 10 & Maxillary premolar-left & Marsupialization (tube drain) & Not erupted & 12 months \\
\hline 5 & M & 11 & Mandibular canine- left & Marsupialization (obturator) & Erupted & 10 months \\
\hline 6 & M & 9 & Mandibular premolar- left Canine- right & Marsupialization (tube drain) & Erupted & 10 months \\
\hline 7 & $\mathrm{~F}$ & 8 & Maxillary premolar- right & Marsupialization (obturator) & Erupted & 8 months \\
\hline 8 & M & 8 & Mandibular 1st molar- bilateral & Marsupialization (tube drain) & Erupted & 7 months \\
\hline 9 & M & 8 & Maxillary premolar- right & Marsupialization (tube drain) & Not erupted & 9 months \\
\hline 10 & M & 7 & Mandibular premolar- left & Marsupialization (tube drain) & Erupted & 9 months \\
\hline 11 & $\mathrm{~F}$ & 7 & Mandibular premolar- right & Marsupialization (obturator) & Erupted & 10 months \\
\hline 12 & M & 12 & Maxillary canine- left & Marsupialization (obturator) & Erupted & 8 months \\
\hline 13 & M & 10 & Mandibular premolar-right & Marsupialization (obturator) & Erupted & 10 months \\
\hline 14 & $\mathrm{~F}$ & 10 & Maxillary molar- left & Marsupialization (obturator) & Erupted & 12 months \\
\hline 15 & M & 9 & Mandibular premolar- right & Marsupialization (obturator) & Erupted & 8 months \\
\hline 16 & $\mathrm{~F}$ & 10 & Maxillary central- right & Marsupialization (obturator) & Erupted & 10 months \\
\hline 17 & $\mathrm{~F}$ & 8 & Maxillary canine- right & Marsupialization (obturator) & Erupted & 11 months \\
\hline
\end{tabular}



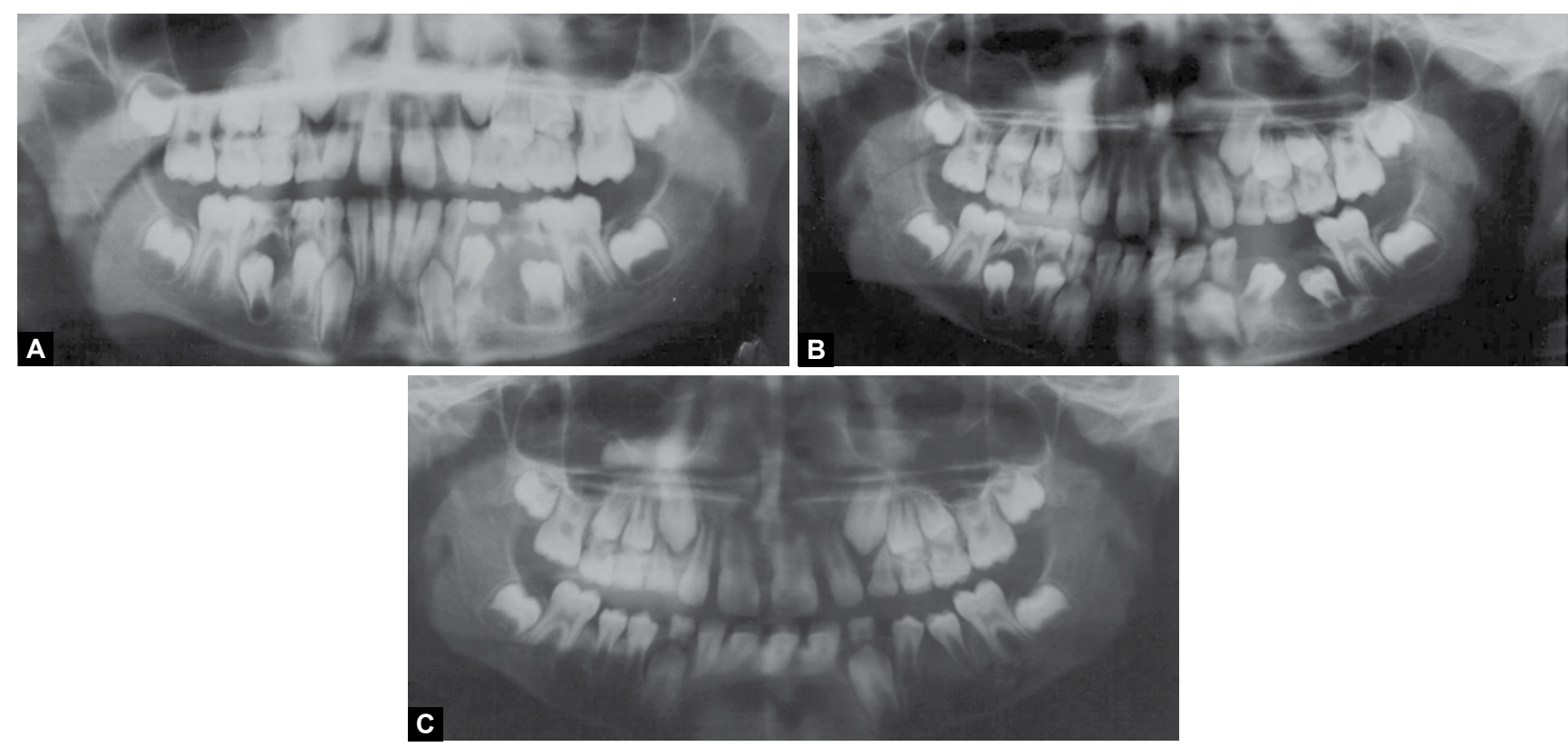

Figs 1A to $\mathrm{C}:(\mathrm{A})$ Preoperative panoramic radiograph of the case, $(\mathrm{B})$ one month after marsupialization, (C) Panoramic radiograph of the case taken at the postoperative 8th month
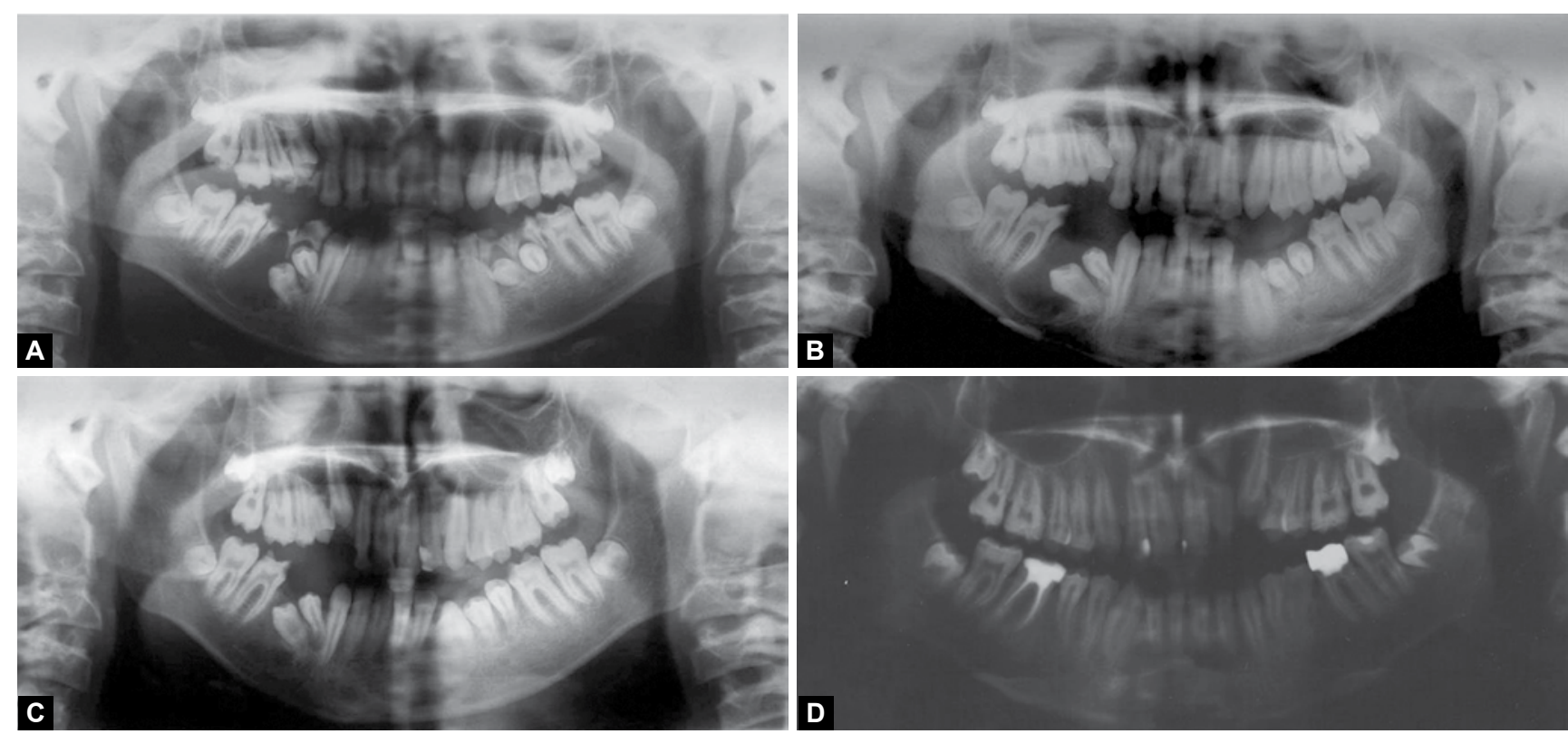

Figs 2A to D: (A) Preoperative panoramic radiograph of an other case, (B) four months after marsupialization, (C) seven months after marsupialization, and (D) panoramic radiograph of the case taken at the postoperative 2 nd year

shows lower morbidity compared to enucleation. ${ }^{4,10}$ On the other hand, a second surgery may be necessary after marsupialization. Nakamura et al reported that marsupialization should be continued for at least 6 months and that surgical removal should be performed if the cyst does not diminish in size. ${ }^{7}$ In addition, because marsupialization does not permit histopathologic examination of the entire cyst epithelium and pathological tissue is left behind, a more aggressive pathological process (i.e. squamous cell carcinoma) may be overlooked. ${ }^{6}$ Marsupialization yielded effective results in 15 patients in the present study, with eruption of the permanent teeth and radiographical examination revealing that the cysts had disappeared. Further, the size of the cysts was reduced and the level of the impacted teeth ascended in two patients; follow-up is ongoing in these patients.

The open cystic cavity is generally filled with iodoform gauze to provide favorable conditions for epithelialization. Following epithelialization, an obturator treatment can be useful in order to prevent food accumulation or closure of the fistula. ${ }^{4}$ In the present study, marsupialization was performed along with obturator treatment in only nine cases, and eight cases were treated by inserting a tube drain into the cystic cavity. The patients were instructed to irrigate the open cavity 3 times a day with an antiseptic solution. Although the tube drain is more hygienic than the obturator and allows irrigation of the cystic cavity that is more effective, its main 

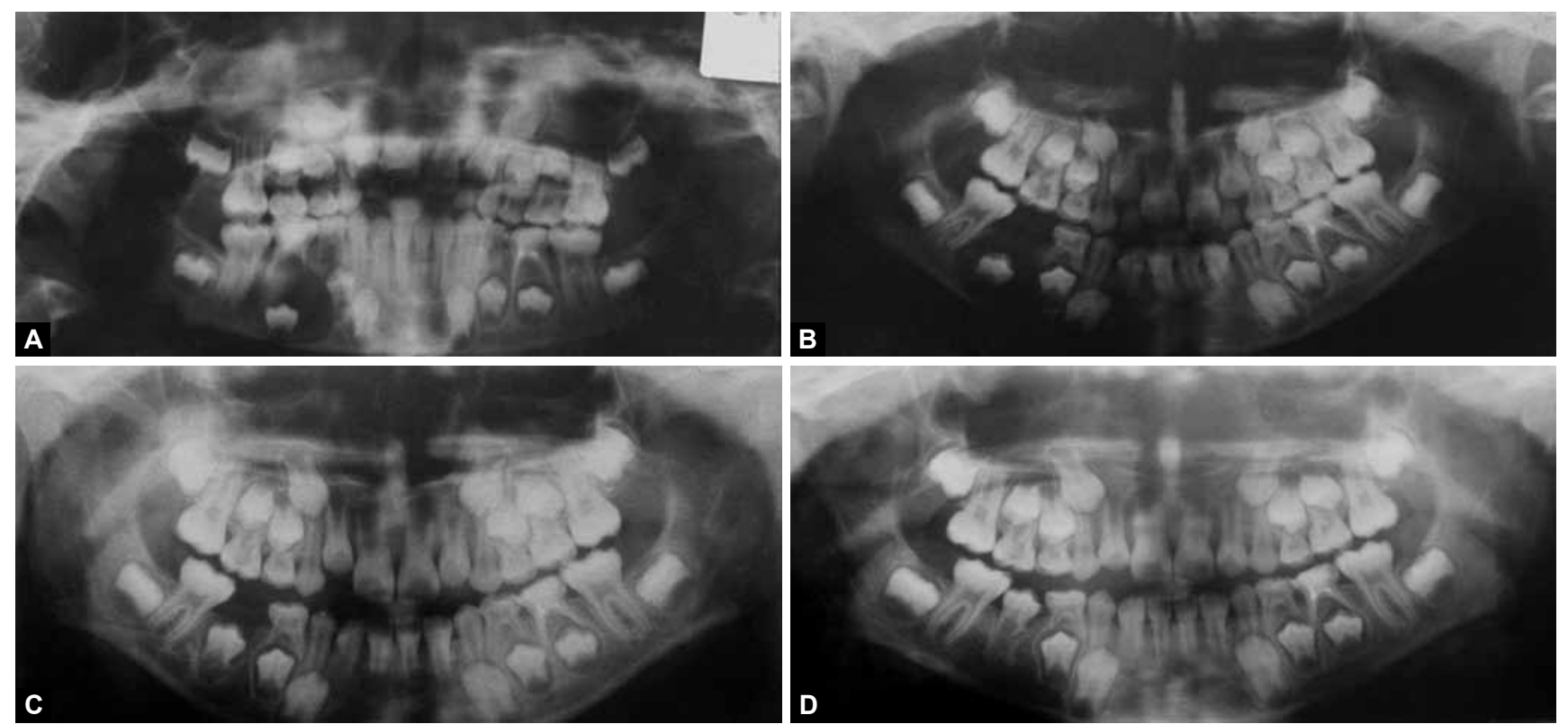

Figs 3A to D: (A) Preoperative panoramic radiograph of the case, (B) one month after marsupialization, (C) six months after marsupialization and (D) panoramic radiograph of the case taken at the postoperative 12th month

disadvantages include difficulty in implanting and stabilizing the tube drain. No differences were observed between acrylic resin obturator and tube drain cases in the present study.

Nakamura et al reported that the effects of marsupialization were more effective in lesions of the mandibular body compared to the mandibular ramus. ${ }^{7}$ Lustig et al reported that nine out of 15 patients with follow-up required adjunctive orthodontic theraphy. ${ }^{14}$ Further, Hyomoto et al reported that marsupialization assisted natural eruption of the impacted tooth in the dentigerous cyst in 42 of 58 subjects $(72.4 \%){ }^{10}$ In our study, tooth eruption with a normal intermaxillary relationship occurred in 7 cases in which the lesion was located in the mandible and in 1 case in which it was located in the maxillae (80\%). Thus, orthodontic therapy was not required. The teeth erupted in the normal position but root formation was not complete in 1 case. The reason for incomplete root development may have been the recurrent infection of the cystic lesion. In addition, the age of the patient (6 years) might have affected the development of the root because root development was not observed radiologically when marsupialization was performed.

Tooth eruption has not been observed in the other 2 cases in which the lesion was located in the maxillae. Although panoramic radiography after marsupialization showed reduction in the size of the cystic cavity, the impacted tooth did not erupt. In one of these cases, the lesion was localized close to the maxillary sinus and infection occurred twice after marsupialization. It is thought that the recurrent infection, the angulation, and the position of the impacted tooth in the alveolar bone may have abated eruption. Orthodontic therapy will be required for the 2 cases in which the lesion was located in the maxillae. We suggest that the most important criteria for orthodontic consultation are the position and the angulation of the impacted teeth. The duration of marsupialization should be changed for each patient, depending on the progress of the individual patient.

\section{CONCLUSION}

Marsupialization should be considered an effective conservative treatment method for jaw cysts that occur during mixed dentition in childhood in order to decrease possible morbidity to the adjacent developing structures. Although marsupialization might be a good treatment option for conservative management of dentigerous cysts, proper case selection is important and long-term follow-up is required.

\section{REFERENCES}

1. Scholl RJ, Kellett HM, Neuman DP, Lurie AG. Cysts and cystic lesions of the mandible: clinical and radiologic-histopathologic review. Scientific Exhibit 1999;19(5):1107-1124.

2. Knezevic G, Jokic D, Knezevic AK. Surgical-orthodontic treatment of developmental odontogenic cysts of the jaws. Acta Stomat Croat 2005;39(1):121-123.

3. Sokler K, Sandev S, Grgurevic J. Surgical treatment of large mandibular cysts. Acta Stomat Croat 2001;35(2):253-257.

4. Sakkas N, Schoen R, Schulze D, Otten JE, Schmelzeisen R. Obturator after marsupialization of a recurrence of a radicular cyst of the mandible. Oral Med Oral Pathol Oral Radiol Endod 2007;103(1):16-18.

5. Marwah N, Bishen KA, Prabha V, Goenka P. A conservative approach in the management of inflammatory dentigerous cyst in transitional dentition: a case report. J Mass Dent Soc 2012; 61(1):18-21.

6. Bodner L. Cystic lesions of the jaws in children. Int J Pediatr Otorhinolaryngol 2002;62(1):25-29.

7. Nakamura N, Mitsuyasu T, Mitsuyasu Y, Taketomi T, Higuchi Y, Ohishi M. Marsupialization for odontogenic keratocysts: longterm follow-up analysis of the effects and changes in growth 
characteristics. Oral Surg Oral Med Oral Pathol Oral Radiol Endod 2002;94(5):543-553.

8. Iatrou I, Theologic-Lygidakis N, Leventis M. Intraosseous cystic lesions of the jaws in children: A retrospective analysis of 47 consecutive cases. Oral Surg Oral Med Oral Pathol Oral Radiol Endod 2009;107(4):485-492.

9. Baykul T, Saglam AA, Aydin U, Başak K. Incidence of cystic changes in radiographically normal impacted lower third molar follicles. Oral Surg Oral Med Oral Pathol Oral Radiol Endod 2005;99(5):542-545.

10. Hyomoto M, Kawakami M, Inoue M, Kirita T. Clinical conditions for eruption of maxillary canines and mandibular premolars associated with dentigerous cysts. Am J Orthod Dentofacial Orthop 2003;124(5):515-520.
11. Delbem AC, Cunha RF, Vieira AE, Pugliesi DM. Conservative treatment of a radicular cyst in a 5-year-old child: a case report. Int J Paediatr Dent 2003;13(6):447-450.

12. Tüzüm MŞ. Marsupialization of a cyst lesion to allow tooth eruption: a case report. Quintessence Int 1997;28(4):283284.

13. Berti Sde A, Pompermayer AB, Couto Souza PH, Tanaka OM, Westphalen VP, Westphalen FH. Spontaneous eruption of a canine after marsupialization of an infected dentigerous cyst. Am J Orthod Dentofacial Orthop 2010;137(5):690-693.

14. Lustig JP, Schwartz-Arad D, Shapira A. Odontogenic cysts related to pulpotomized deciduous molars clinical features and treatment outcome. Oral Surg Oral Med Oral Pathol Oral Radiol Endod 1999;87(4):499-503. 